\title{
Analysis Methods for Neutrino Follow-up Observations with MAGIC
}

\author{
Alicia Fattorini ${ }^{* 1}$, K. Satalecka ${ }^{2}$, E. Bernardini ${ }^{3}$, A. Berti ${ }^{4}$, J. Bolles ${ }^{1}$, P. Gliwny ${ }^{5}$ \\ for the MAGIC Collaboration ${ }^{\dagger}$ \\ 1) Technische Universität Dortmund, Dortmund, Germany \\ 2) Deutsches Elektronen-Synchrotron (DESY) Zeuthen, Zeuthen, Germany \\ 3) Università degli studi di Padova, Padova, Italy \\ 4) University of Torino and INFN Torino, Torino, Italy \\ 5) University of Lodz, Lodz, Poland \\ E-mail: alicia.fattorinietu-dortmund.de
}

With the first measured correlation of an extremely high-energy neutrino event and a flaring gamma-ray source in September 2017, multimessenger astronomy has become more important than ever. The stereoscopic IACT system MAGIC, located at La Palma, Canary Islands, is involved in neutrino follow-up campaigns since 2012. The MAGIC telescopes are sensitive for gamma events with energies from the $\sim 30 \mathrm{GeV}$ range up to tens of $\mathrm{TeV}$. When a potential astrophysical neutrino is detected by IceCube, an alert with the reconstructed coordinates is published. MAGIC, making use of an automated alert response system, performs follow-up observations in search of a correlated gamma-ray flux. The reconstructed neutrino direction is given with an uncertainty, typically around $0.2^{\circ}-1^{\circ}$. As the angular resolution of MAGIC is much smaller, the analysis for discovering sources in a given region has to be modified. In case of a non detection, in order to interpret the data correctly, an information about flux upper limits in the whole IC event error region should be given. Here we present a method to produce sky maps for identifying point sources or deriving flux upper limits on the desired sky region, based on a maximum likelihood approach included in the SkyPrism software. Examples of results from MAGIC observations of IC-170922A and other IceCube alerts will be shown.

36th International Cosmic Ray Conference -ICRC2019-

July 24th - August 1st, 2019

Madison, WI, U.S.A.

\footnotetext{
* Speaker.

†https://magic.mpp.mpg. de/. For collaboration list see PoS(ICRC2019)1177
} 


\section{Multimessenger Astronomy}

With the first detection of high-energy, most likely astrophysical neutrinos at the IceCube observatory in 2013 [1], a new era of multimessenger astronomy has began. In order to investigate the origin of cosmic neutrinos and search for counterparts with other detectors, the Astronomical Multimessenger Observatory Network (AMON) [2] with an alert system was founded. Among others, the MAGIC telescopes are involved in follow-up campaigns to search for a counterpart in the very high-energy gamma-ray band.

\subsection{The Instruments}

The Major Atmospheric Gamma-ray Imaging Cherenkov (MAGIC) telescopes form a stereoscopic Imaging Air Cherenkov Telescope system, located at the Canary Island La Palma at $2200 \mathrm{~m}$ above sea level. The detector is sensitive to gamma photons with energies between $\sim 30 \mathrm{GeV}$ and up to tens of TeVs. The mirror surface of each telescope has a diameter of $17 \mathrm{~m}$, focusing the light to 1039 Photo Multiplier Tubes (PMTs). The field of view of MAGIC is $3.5^{\circ}$, the angular resolution reaches $0.1^{\circ}$. With its lightweight carbon fiber structure, MAGIC was designed to perform extremely fast repositioning maneuver for follow-up observations of transient sources. Nowadays, MAGIC is able to turn $180^{\circ}$ in $27 \mathrm{~s}$ in the fast mode, reaching a high speed of $7^{\circ}$ per second. [3]

With its volume of $1 \mathrm{~km}^{2}$ the IceCube detector is currently the biggest neutrino detector. It is located at the South Pole and consists of 86 strings with 80 Digital Optical Modules (DOMs) each. The PMTs, installed in the DOMs, measure the Cherenkov light, caused by relativistic charged secondary particles, which are produced in neutrino interactions with the Antarctic ice. [1]

While a general astrophysical neutrino flux has long been detected by IceCube, the point source identification turns out to be extremely challenging, because of the limited angular resolution and the relatively low statistics of likely astrophysical neutrino events. [4]

\subsection{The Alert System}

Since 2012 MAGIC has been involved in neutrino follow-up campaigns. When IceCube detects a likely astrophysical neutrino event, the alert reaches the main data center in Wisconsin, Madison, with a delay of $\sim 33 \mathrm{~s}[5]$ after the event. The coordinates and further information are published via the Gamma-ray Coordinates Network (GCN) [6] as well as the AMON to make the alert available for all observatories. The fast data transfer of the network and the high repositioning speed of MAGIC make follow-up observations in the electromagnetic high-energy band $\sim 1$ min after a neutrino event possible. Since the first published alert in 2016, around 6 alerts per year are sent out by IceCube. The with the updated system (June 2019) the rate of expected alerts increases to $\sim 10$ per year [7].

\section{The SkyPrism Likelihood Analysis}

In the case of neutrino alerts, the reconstructed coordinates of the events are given with a relatively large uncertainty of typically $\sim 1^{\circ}$ at a $90 \%$ confidence level. The main task of the data analysis is the search of a gamma-ray source within the given uncertainty region, which is usually covered by the field of view of MAGIC. 
SkyPrism is an analysis tool of MAGIC, based on a maximum likelihood approach from the EGRET analysis in [8]. Originally the software was written for the analysis of extended gammaray sources, but it can be applied on various cases, like searching for point sources within a certain region. With the assumption of the source properties and the instrument response of MAGIC a model can be created, described by a likelihood function. Among others, the number of gamma events from the source is a free parameter of the likelihood. With a maximum likelihood test the values for those parameters that describe the real data best are calculated. [9]

\subsection{The Point Source Search}

In the context of point source search with SkyPrism, the neutrino uncertainty region is scanned by testing a point source model on each position of a predefined grid. The resulting test statistic (TS) value gives information about the probability of the existence of a gamma-ray source at each specific position. The advantage of this analysis is the minimized computing time and power, since the parameters for the models are calculated in a sky map representation, so the source model is obtained by adjusting the coordinates of the assumed grid positions (see fig. 1).

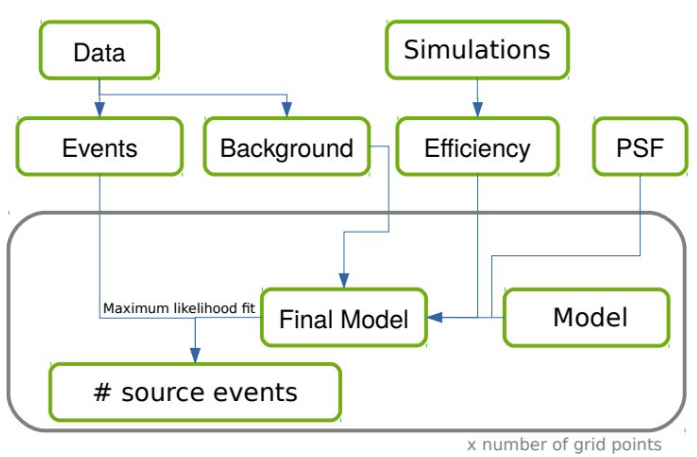

Figure 1: Overview of creating sky maps with SkyPrism testing the point source model at every grid position of the sky map.

\subsection{Evaluation of the Analysis}

Since SkyPrism is especially tested for sources with known coordinates, additional checks for the case of point source searching within certain regions in the context of follow-up observations are
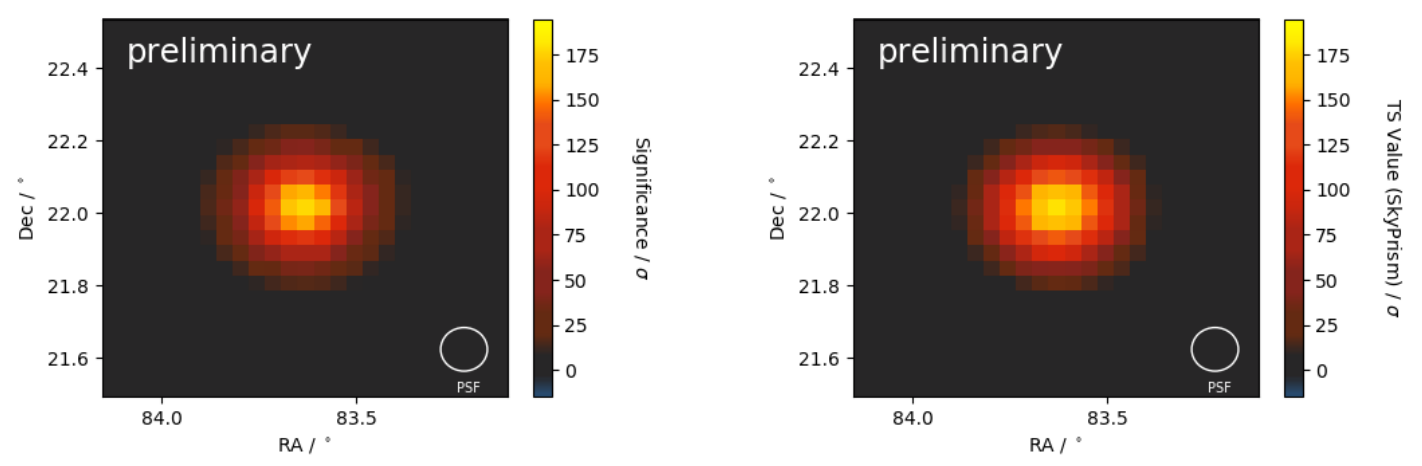

Figure 2: Sky map of the Crab Nebula calculated with the standard software MARS (left) and the sky map produced with SkyPrism (right).

necessary. A reliable way to check a new analysis method is testing it with a strong and well-known source. The strongest stable source known in the energy range of MAGIC is the Crab Nebula, a pulsar wind nebula in the Milky Way. For the check, a data set of $\sim 16 \mathrm{~h}$ of observation time at low zenith distances $<35^{\circ}$ and good weather conditions is used. As a test for the point source searching following section 2.1, a TS value map of the Crab Nebula is produced. The standard 


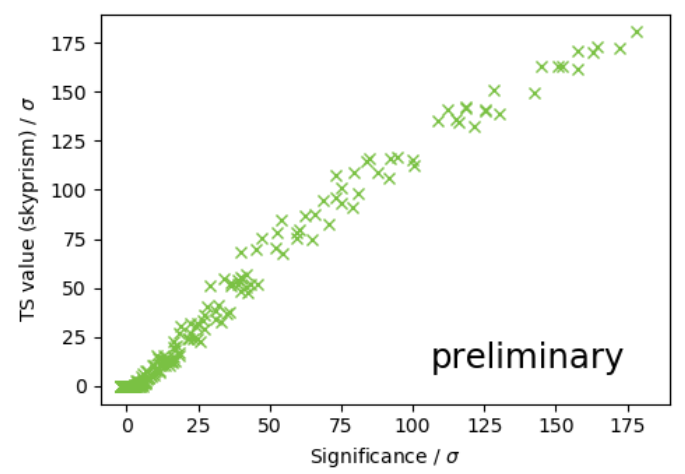

Figure 3: Correlation of significance (MARS) and TS value (SkyPrism) within the sky map. software of MAGIC, called MARS [10], also offers a tool for sky maps, using the Li \& Ma method [11] to calculate the significances of possible sources in a certain region. The sky maps are shown in fig. 2. For the direct comparison, a correlation plot (see fig. 3) is produced. The plot shows a high correlation between the MARS significance and the SkyPrism TS value, which means that both analyses lead to comparable results over the whole region and are capable to detect bright sources significantly.

\subsection{Flux Maps with SkyPrism}

For the physical interpretation of the data, the flux of a potential source is calculated. The flux is given by the number of gamma particles from the source per second per area. To obtain the number of source events from the number of measured gamma events, the efficiency of the detector has to be well-known. In the case of MAGIC the Instrument Response Function (IRF) is calculated by the simulation of gamma events, their propagation through the atmosphere and the detector acceptance. The upper limits of the number of gamma events from the source and thus the flux upper limit is calculated at a $95 \%$ confidence level. The IRFs are strongly dependent on the energy of the primary particle. In this work, the analysis is done in a single energy range. Therefore, the energy threshold for the flux calculation is selected so that the IRF remain welldescribed throughout the entire energy range.

\section{Neutrino Follow-up Observations by MAGIC}

In the case of a neutrino alert via the GCN channel, MAGIC uses its automated alert response system [12], which checks general observation conditions of the reconstructed coordinates at the telescopes site and initiates the follow-up observation of the published position. MAGIC performed six neutrino follow-up observations, marked in fig. 4. The flare of the source TXS 0506+056 measured by MAGIC in 2017 was spatially correlated with the neutrino event EHE-170922 [13] detected by IceCube a few days

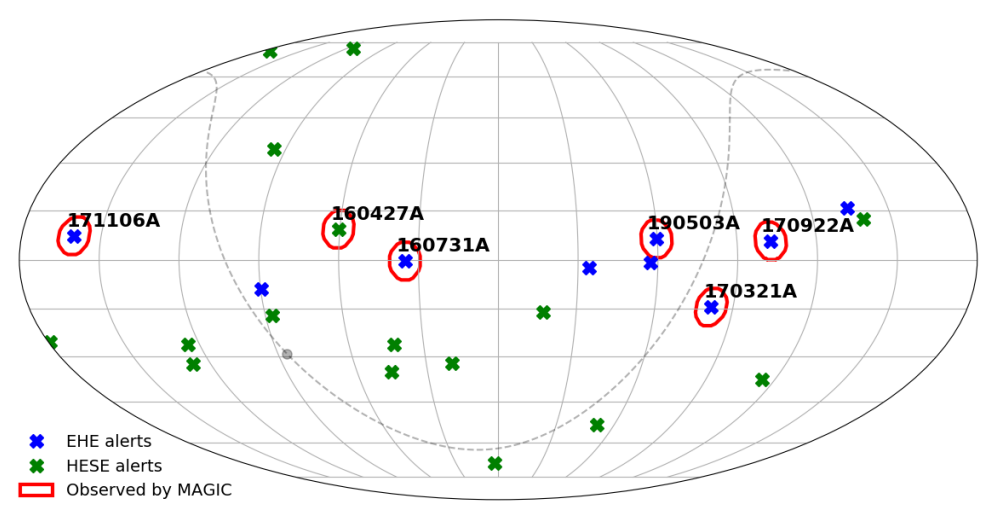

Figure 4: Sky map of the coordinates of all IceCube alerts. [6] 
before the gamma flare. The TS value maps as well as the flux maps of tree follow-up observations are shown in the next chapters: the first follow-up observation of the neutrino event called HESE-160427A in 2016, the most recent one of EHE-190503A and the analysis results of the data of MAGIC observations of the blazar TXS 0506+056, a likely counterpart of the EHE-170922A alert.

\subsection{First Neutrino Follow-up Observation: HESE-160427A}

The first publication of a neutrino event from the IceCube collaboration via the GCN and AMON channels was on the 27th of April 2016. Because of full moon, MAGIC started the observation with a delay of two days. The measurement was carried out for two hours with moderate moon conditions at low zenith distances $<30^{\circ}$ and clear sky. The sky maps of the SkyPrism software are shown in fig. 5. The fluctuations in both maps are comparable. Although the TS value
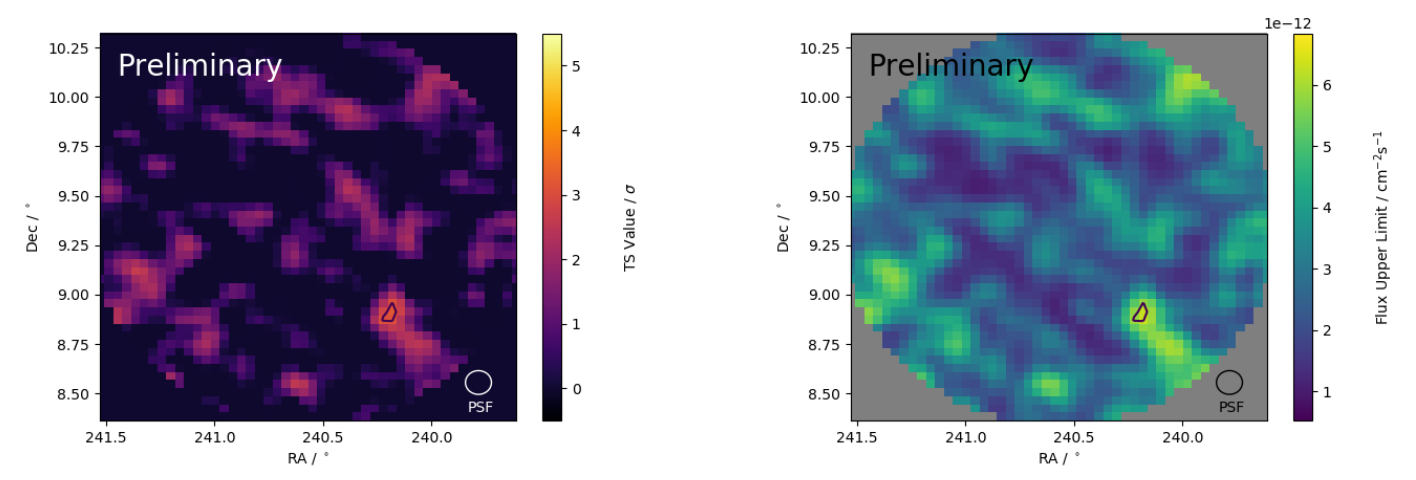

Figure 5: TS value map (left) and upper limit flux map (right) of HESE-160427A.

map does not provide the detection of a possible neutrino source within the uncertainty region, the flux upper limit skymap shows consistent results with the TS value map and makes a physical interpretation of the measurement possible. A potential neutrino source with the given assumptions can be limited by an integrated flux $(>300 \mathrm{GeV})$ of $6.3 \times 10^{-12} \mathrm{~cm}^{-2} \mathrm{~s}^{-1}$ with a maximum TS value of $3.2 \sigma$ within the analyzed sky region.

\subsection{First Source Detection in IceCube Region: EHE-170922A/TXS 0506+056}

On the 22th of September 2017 the IceCube Collaboration detected a muon neutrino with a reconstructed energy of $\sim 290 \mathrm{TeV}$ [13] and the alert was published with a delay of $43 \mathrm{~s}$ [13] via the GCN/AMON channel. The best-fit coordinates from IceCube are right ascension (RA) $77.43_{-0.65}^{+0.95}$ and declination (Dec) $5.72_{-0.30}^{+0.50}$ (in degress, J2000 equinox, $90 \%$ ) [13]. MAGIC performed the follow-up measurements on the 24th of September 2017, but no detection was archived during more than one hour observation. A second set of observations was made from the 28th of September until the 4th of October, observing TXS 0506+056, a blazar spatially coincident with the reconstructed neutrino position and, according to the Fermi-LAT announcement of the 28th of September [14], in a flaring state. During these days MAGIC was able to collect data in $\sim 13$ hours of observation time. The source was observable at a zenith distance between $22^{\circ}$ and $52^{\circ}$ and good weather conditions. It was finally discovered by MAGIC in the very high-energy gamma rays above $90 \mathrm{GeV}$ [15]. 
In fig. 6 the analysis of the second data set, equal to the data set in [13], with the SkyPrism software is shown. The contours show the $90 \%$ (red) and the $50 \%$ (gray) uncertainty region of the neutrino event. The TS value, calculated at the position of TXS $0506+056$, reaches $6.2 \sigma$ with the maximum likelihood method of SkyPrism. This result is consistent with the significance calculated with the MAGIC standard analysis and presented in the paper [13].

For the calculation of the integrated flux

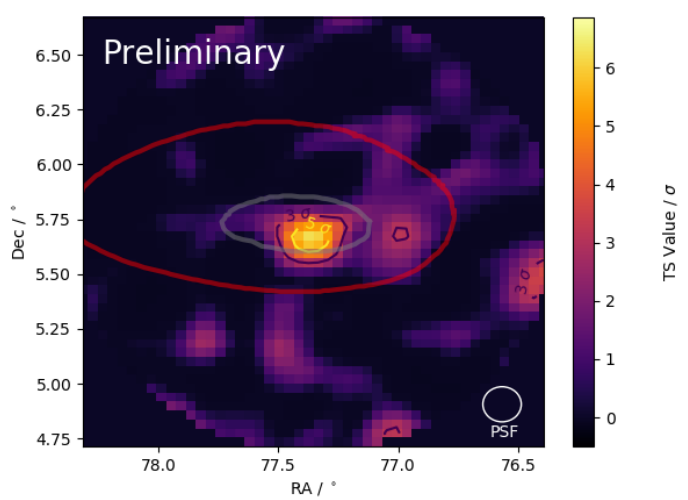

Figure 6: TS value map of EHE-170922A. map, an energy threshold of $300 \mathrm{GeV}$ is chosen. The resulting TS value and flux map are shown in fig. 7. At higher energies the source is less significant, the maximum TS value is $3.7 \sigma$ and
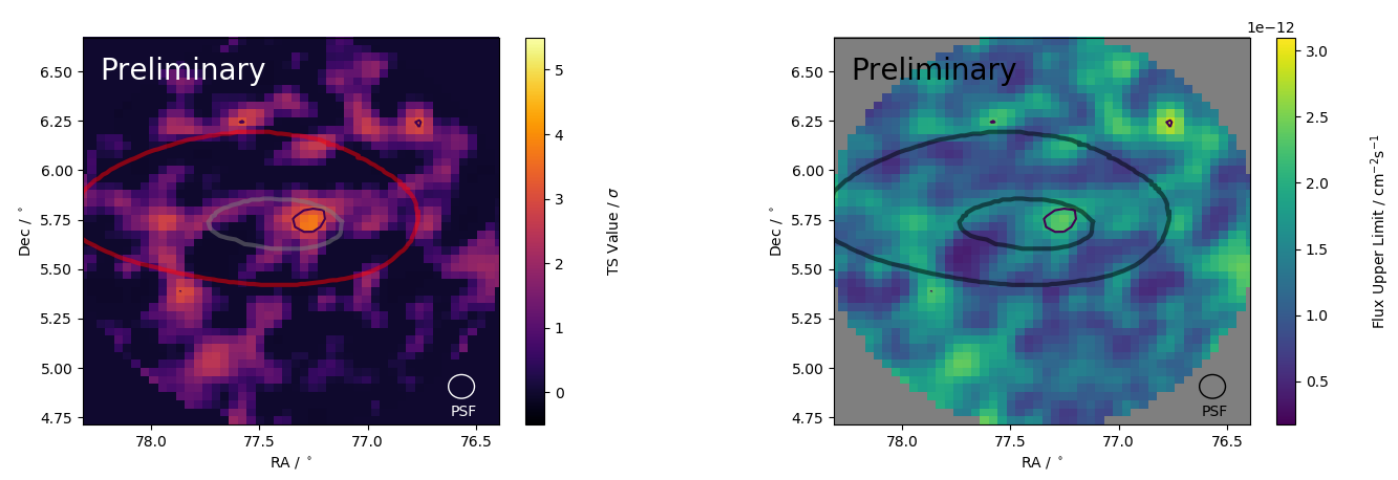

Figure 7: TS value map (left) and upper limit flux map (right) of EHE-170922A.

the flux can be limited by $2.9 \times 10^{-12} \mathrm{~cm}^{-2} \mathrm{~s}^{-1}$. The flux is calculated as an average flux over the observation time. Regarding that the flux was strongly variable during those days, a temporally binned analysis, see [16], is recommended.

\subsection{Neutrino Follow-up Observation in 2019: EHE-190503A}

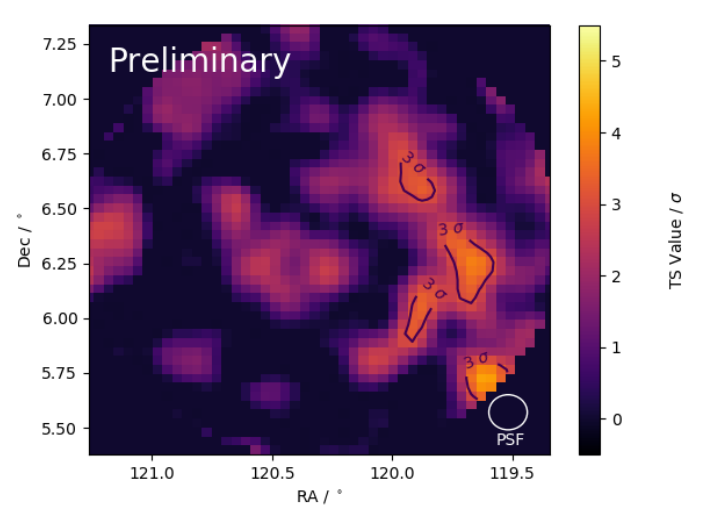

Figure 8: TS value map of EHE-190503A.
On the 3rd of May 2019 the IceCube Collaboration published a neutrino alert via the GCN and AMON channel. The position was observable for the MAGIC telescopes in the beginning of the following night at a zenith distance between $44^{\circ}$ and $52^{\circ}$ and good weather conditions. The reconstructed neutrino position was observed for $30 \mathrm{~min}$ in total. In fig. 8 the TS value map at low energies $(>90 \mathrm{GeV})$ is shown. The maximum TS value is $4.3 \sigma$ within the region. 
No detection of a source can be claimed within the IceCube uncertainty region at this energy range. The flux upper limit map is calculated at higher energies $(>300 \mathrm{GeV})$ to ensure stable IRFs in the given energy range, and is shown in fig. 9 as well as the corresponding TS value map.found No
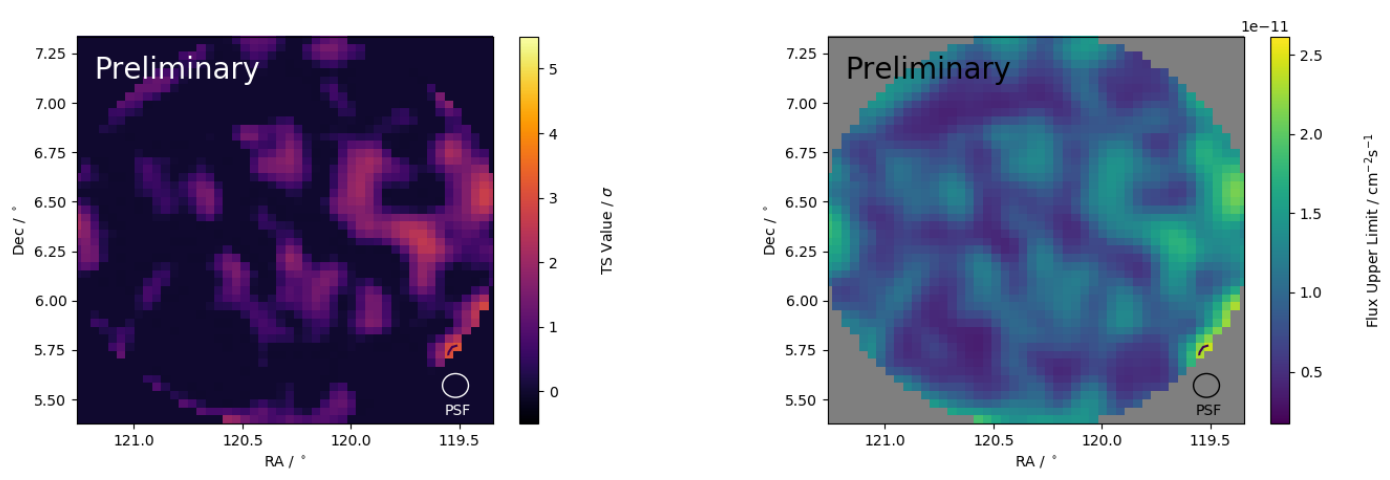

Figure 9: TS value map (left) and upper limit flux map (right) of EHE-190503A.

source was detected at higher energies neither. The maximum TS value within the analyzed region is $3.2 \sigma$. The integrated flux of a potential gamma-ray source within the IceCube uncertainty region can be limited to $2.4 \times 10^{-11} \mathrm{~cm}^{-2} \mathrm{~s}^{-1}$ at energies above $300 \mathrm{GeV}$.

\section{Summary and Outlook}

In the field of multimessenger astronomy, analysis methods must be adapted to new requirements. The maximum likelihood method is very well suited for the search for point sources within the uncertainty region of IceCube follow-up observations. Additional tests are required to check whether the maximum likelihood analysis can achieve a gain in sensitivity. The new method was tested on three neutrino alerts from IceCube. The results are compatible with the ones obtained with the standard software of MAGIC. In particular the detection of TXS0506+056 can be reproduced with SkyPrism. The TS value of $6.2 \sigma$ calculated with the maximum likelihood analysis is equal to the significance calculated with the current analysis tools of MARS and published by the MAGIC Collaboration. The integrated flux can be limited by $2.9 \times 10^{-12} \mathrm{~cm}^{-2} \mathrm{~s}^{-1}$ for energies above $300 \mathrm{GeV}$.

Further plans are the implementation of an energy binned analysis for flux upper limit calculations and the corresponding TS value over a given energy range to consider the energy dependence of the different parameters. A second goal is to implement the calculation of systematic uncertainties of the flux upper limits. With the new analysis method for the point source search, the MAGIC collaboration is optimally prepared for future neutrino alerts. 


\section{References}

[1] IceCube Collaboration, JINST 12 (2017) P03012.

[2] M. W. E. Smith et al., Astropart. Phys. 45 (2013) 56-70.

[3] MAGIC Collaboration, Astropart. Phys. 72 (2016) 76-94.

[4] IceCube Collaboration, M. G. Aartsen et al., Eur. Phys. J. C79 (2019) 234.

[5] IceCube Collaboration, Astropart. Phys. 92 (2017) 30-41.

[6] HEASARC, Astrophysics Science Division (ASD), NASA’s GSFC., "Gcn: The gamma-ray coordinates network."

[7] IceCube Collaboration, PoS ( ICRC2019) 1021 (these proceedings).

[8] EGRET Collaboration, J. R. Mattox et al., Astrophys. J. 461 (1996) 396.

[9] I. Vovk, M. Strzys, and C. Fruck, Astron. Astrophys. 619 (2018) A7.

[10] MAGIC Collaboration, R. Zanin, PoS ( ICRC2013) 0773 (2013).

[11] T.-P. Li and Y.-Q. Ma, The Astrophysical Journal 272 (1983) 317-324.

[12] MAGIC Collaboration, A. Berti et al., POS ( ICRC2019) 633 (these proceedings).

[13] IceCube, Fermi-LAT, MAGIC, AGILE, ASAS-SN, HAWC, H.E.S.S., INTEGRAL, Kanata, Kiso, Kapteyn, Liverpool Telescope, Subaru, Swift NuSTAR, VERITAS, VLA/17B-403

Collaboration, M. G. Aartsen et al., Science 361 (2018) eaat1378.

[14] Fermi-LAT Collaboration, Y. T. Tanaka, S. Buson, and D. Kocevski, The Astronomer's Telegram 10791 (Sep, 2017) 1.

[15] MAGIC Collaboration, R. Mirzoyan, The Astronomer's Telegram 10817 (Oct, 2017) 1.

[16] MAGIC Collaboration, S. Ansoldi et al., Astrophys. J. Lett. (2018). 